\title{
Perfluoroalkyl substances in soils around the Nepali Koshi River: levels, distribution, and mass balance
}

\author{
Bing Tan • Tieyu Wang • Pei Wang • Wei Luo • \\ Yonglong Lu • Kumar Y. Romesh • John P. Giesy
}

Received: 24 January 2014 / Accepted: 24 March 2014 / Published online: 8 April 2014

(C) Springer-Verlag Berlin Heidelberg 2014

\begin{abstract}
Perfluoroalkyl substances (PFASs) were analyzed in surface soils along the Koshi River in Nepal, a typical agricultural country with little industrialization and urbanization. Sixteen target PFASs were quantified in soils from a hilly region in central and eastern Nepal, but only ten PFASs were detected. Concentrations of total PFASs ranged from nd (below the detection limit) to $1.78 \mathrm{ng} / \mathrm{g} \mathrm{dw}$. The predominant PFASs in soils were perfluoro-octanoic acid (PFOA) and perfluoro-butanesulfonate (PFBS) with concentrations that ranged from nd to $0.26 \mathrm{ng} / \mathrm{g} \mathrm{dw}$ and nd to $0.38 \mathrm{ng} / \mathrm{g} \mathrm{dw}$, respectively. Results of mass balance analysis also revealed weak associations among concentrations of PFASs, extractable organic fluorine (EOF), and total fluorine (TF). PFASs were relatively evenly distributed among locations. Due to the absence of direct emission of PFASs and slow development of local industry, PFASs in soils originated mostly from longrange atmospheric transport, consumer use, and disposal of
\end{abstract}

Responsible editor: Ester Heath

Electronic supplementary material The online version of this article (doi:10.1007/s11356-014-2835-6) contains supplementary material, which is available to authorized users.

B. Tan $\cdot$ T. Wang $(\bowtie) \cdot$ P. Wang $\cdot$ W. Luo $\cdot$ Y. Lu

State Key Lab of Urban and Regional Ecology, Research Center for Eco-environmental Sciences, Chinese Academy of Sciences,

Beijing 100085, China

e-mail: wangty@rcees.ac.cn

B. Tan

College of Environmental Science and Engineering, Liaoning

Technical University, Fuxin 123000, China

K. Y. Romesh

Department of Environmental Science and Engineering, School of science, Kathmandu University, Kathmandu 6250, Nepal

J. P. Giesy

Department of Veterinary Biomedical Sciences and Toxicology

Centre, University of Saskatchewan, Saskatoon, Canada
PFASs-containing products. Uncontrolled disposal of domestic waste will be a challenge to controlling concentrations of PFASs in Nepal.

Keywords PFASs $\cdot$ Soil $\cdot$ Land use $\cdot$ Mass balance . The Koshi River

\section{Introduction}

Perfluoroalkyl substances (PFASs), as a class of emerging persistent organic pollutants (POPs), have been widely used since they were first manufactured in the 1950s. Because of their unique properties such as high surface activity, thermal and acid resistance, and repellency of both water and oil, PFASs have been used in various industries such as surfactants and surface protectors in carpets, leather, paper, food packaging, textile coating, upholstery, fire-fighting foams, floor polishes, and shampoos (Giesy and Kannan 2001, 2002; Xie et al. 2013). The high stability of carbonfluorine bonds renders PFASs resistant to hydrolysis, photolysis, microbial degradation, and metabolism by vertebrates (Kissa 2001). Because of their low solubility in water and negligible vapor pressure when dissolved in water (low Henry's constant) (US-EPA 2013), most PFASs can accumulate in aquatic systems and transported by hydrological processes (Hansen et al. 2002; Yamashita et al. 2005), while PFASs in soils can also be transported to the atmosphere, surface water, and groundwater through volatilization, diffusion, leaching, and mass flow (Armitage et al. 2009), which results in bioaccumulation in food chains in aquatic fauna and environmental deterioration. These chemical features coupled with the increased use of PFASs have led to environmental threats of bioaccumulation and land and water contamination (Pico et al. 2011). 
PFASs have been widely introduced into different environmental media and different regions including remote areas (Dreyer et al. 2009; Karrman et al. 2007; Young et al. 2007). Therefore, the issue of adverse impacts on ecosystems and health of humans caused by PFSAs is still of long-term concerns. Since the addition of PFOS and its salts were added to Annex B of the Stockholm Convention on Persistent Organic Pollutants (UNEP) in 2009, Europe, North America, and Japan have legislated so that production of chemicals containing PFOS is eliminated or curtailed (Wang et al. 2009). To meet the growing demand for surfactants and other surface modification applications, alternatives were already developed to replace PFOS and uses of homologues including perfluorobutanesulfonate (PFBS) and perfluorobutanoic acid (PFBA) in some industries (Oliaei et al. 2013).

Nepal is a landlocked sovereign state located on the MidHimalayan mountain section of Asia, with an area of $147,181 \mathrm{~km}^{2}$ and a population of approximately 27 million (NPHC 2012). Nepal shares a border with the People's Republic of China to the north, and the Republic of India to the south, east, and west. It is a mountainous country with about $75 \%$ of its total area consisting of mountains, hills, elevated plains, and river valleys. Due to the slow development of the economy, to date, the Nepalese Government has not fully developed its abundant natural resources, such as the forest, water, and minerals. Agriculture is the dominant economic activity of the Nepalese people. Crops like paddy, maize, millet, and potato are widespread in this region. Most of the existing industries in Nepal are agro based and forest based, and include: jute, sugar, textiles, leather shoes, tobacco, plastic, and so on (Shrestha 2005).

Nepal is faced with increasing environmental issues. Organochlorine pesticides (OCPs), polycyclic aromatic hydrocarbons (PAHs), hexachlorobenzene (HCB), polychlorinated biphenyls (s), polybrominated diphenyl ethers (PBDEs), and heavy metals are present in various environmental media in Nepal (Aichner et al. 2007; Guzzella et al. 2011; Bhatt and Gardner 2009; Dahal et al. 2008) (Table S1). However, previous studies have focused on classic persistent toxic substances (PTS), and little information is available for emerging PTS such as PFASs. Nevertheless, there is evidence to show the existence of PFASs in fishes in the headstream region of the Koshi River (Shi et al. 2010). PFASs distributions in water and biota were also reported in the Ganges River Basin, which is a downstream area of Koshi River (Corsolini et al. 2012; Yeung et al. 2009a). Thus, it was deemed important to examine whether the Koshi River segment in Nepal is also contaminated by PFASs. The present study was designed to determine whether PFASs exist in the Nepali environment and to determine the distribution and fate of PFASs under such warm and humid monsoon climate and high altitude. Since soils serve as an important sink of PTS in pollution cycling they have a large capacity to retain contaminants and can also emit POPs into the atmosphere. The present study was conducted as a systematic investigation to trace sources and fates of toxic substances in various environmental media from the Tibetan Plateau. The objectives were to determine concentrations and distribution of PFASs in soils around a main watershed of Nepal, and to identify the potential sources and provide information for future management and soil remediation. This study would help to establish baseline information of PFASs in Nepalese environment. To our knowledge, this investigation represents the first published data on PFASs in Nepal and also one of the few studies on PFASs in soils.

\section{Materials and method}

Standards and reagents

HPLC grade methanol, methyl tert-butyl ether (MTBE), tetrabutylammonium hydrogen sulfate (TBAHS), and ammonium acetate were purchased from J.T. Baker (Phillipsburg, NJ, USA). Analytical grade sodium thiosulfate was purchased from EMD Chemicals (Gibbstown, NJ, USA). Nano-pure water was obtained from a Milli-Q gradient A-10 (Millipore, Bedford, MA, USA). Supelclean ENVI-Carb (120-400 mesh, $100 \mathrm{~m}^{2} / \mathrm{g}$ ) was purchased from Sigma-Aldrich Co. (St. Louis, MO, USA). Mixtures of 17 native and 11 mass-labeled $\left({ }^{13} \mathrm{C}\right.$ or ${ }^{18} \mathrm{O}$ ) perfluoroalkyl acids (PFAAs) standards, and individual native and mass-labeled $\left({ }^{13} \mathrm{C}\right.$ or $\left.{ }^{2} \mathrm{H}\right)$ standards of 3 precursors were purchased from Wellington Laboratories, with purities of $>98 \%$ (Guelph, Ontario, Canada). Sixteen PFASs were quantified in this study (Table 1).

\section{Collection of samples}

Surface soils were collected along the watershed of the Koshi River, which is one of the largest tributaries of the Ganges River and locates in central and eastern Nepal. These regions are comparatively more developed than other regions in Nepal, and contain more trade centers, major industrial distribution centers, booming domestic tourism, as well as larger populations. Fourteen surface soil samples were collected along this watershed in April, 2010 using a stainless steel trowel (Fig. 1). Each sample was prepared by mixing five sub-samples from an area of about $10 \times 10 \mathrm{~m}$. Samples were transferred and stored in clean polypropylene (PP) bags. Duplicates were collected daily, and analyzed along with laboratory and procedural blanks. Soil samples were transported to the laboratory, air-dried and ground and homogenized with a silica mortar and pestle after discarding large stones, plant and animal residues, and then sieved 
Table 1 Detection limits, recoveries, and detection rates of PFASs

${ }^{\text {a }}$ Number of samples detected

\begin{tabular}{|c|c|c|c|c|}
\hline Analyte & Acronym & $\begin{array}{l}\text { LOD } \\
(\mathrm{ng} / \mathrm{g})\end{array}$ & $\begin{array}{l}\text { Recovery } \\
(\%)\end{array}$ & $\begin{array}{l}\text { Detection } \\
\text { rate }^{\mathrm{a}}(\%)\end{array}$ \\
\hline Perfluorocarboxylic acid & PFCAs & & & \\
\hline Perfluoro-butanoic acid & PFBA (C4) & 0.21 & $76 \pm 7$ & $4(29)$ \\
\hline Perfluoro-pentanoic acid & PFPeA (C5) & 0.03 & $94 \pm 11$ & $2(14)$ \\
\hline Perfluoro-hexanoic acid & PFHxA (C6) & 0.09 & $83 \pm 5$ & $5(36)$ \\
\hline Perfluoro-heptanoic acid & PFHpA (C7) & 0.24 & $96 \pm 10$ & $1(7)$ \\
\hline Perfluoro-octanoic acid & PFOA (C8) & 0.11 & $89 \pm 3$ & $8(57)$ \\
\hline Perfluoro-nonanoic acid & PFNA (C9) & 0.09 & $92 \pm 5$ & $0(0)$ \\
\hline Perfluoro-decanoic acid & PFDA (C10) & 0.09 & $86 \pm 4$ & $0(0)$ \\
\hline Perfluoro-undecanoic acid & PFUdA (C11) & 0.15 & $88 \pm 8$ & $0(0)$ \\
\hline Perfluoro-dodecanoic acid & PFDoA (C12) & 0.09 & $92 \pm 7$ & $1(7)$ \\
\hline Perfluorinated sulfonic acid & PFSAs & & & \\
\hline Perfluoro-butanesulfonate & PFBS (C4) & 0.09 & $76 \pm 9$ & $6(43)$ \\
\hline Perfluoro-hexanesulfonate & PFHxS (C6) & 0.30 & $94 \pm 8$ & $0(0)$ \\
\hline Perfluoro-octanesulfonate & PFOS (C8) & 0.09 & $97 \pm 9$ & $2(14)$ \\
\hline Perfluoro-decanesulfonate & PFDS (C10) & 0.21 & $95 \pm 11$ & $0(0)$ \\
\hline \multicolumn{5}{|l|}{ Percursors } \\
\hline Perfluoro-1-octanesulfonamide & FOSA & 0.10 & $107 \pm 12$ & $0(0)$ \\
\hline $\begin{array}{l}\text { 2- } N \text {-methylperfluoro-1- } \\
\text { octanesulfonamido-ethanlo }\end{array}$ & $N$-MeFOSE & 0.24 & $85 \pm 6$ & $1(7)$ \\
\hline $\begin{array}{l}\text { 2- } N \text {-ethylperfluoro-1- } \\
\text { octanesulfonamido-ethanlo }\end{array}$ & $N$-EtFOSE & 0.27 & $75 \pm 8$ & $3(21)$ \\
\hline \multicolumn{5}{|l|}{ International standards } \\
\hline${ }^{13} \mathrm{C}_{4}$ Perfluoro-butanoic acid & ${ }^{13} \mathrm{C}_{4}$ PFBA & - & - & - \\
\hline${ }^{13} \mathrm{C}_{4}$ Perfluoro-hexanoic acid & ${ }^{13} \mathrm{C}_{4} \mathrm{PFHxA}$ & - & - & - \\
\hline${ }^{13} \mathrm{C}_{4}$ Perfluoro-octanoic acid & ${ }^{13} \mathrm{C}_{4}$ PFOA & - & - & - \\
\hline${ }^{13} \mathrm{C}_{4}$ Perfluoro-nonanoic acid & ${ }^{13} \mathrm{C}_{4}$ PFNA & - & - & - \\
\hline${ }^{13} \mathrm{C}_{4}$ Perfluoro-decanoic acid & ${ }^{13} \mathrm{C}_{4}$ PFDA & - & - & - \\
\hline${ }^{13} \mathrm{C}_{4}$ Perfluoro-undecanoic acid & ${ }^{13} \mathrm{C}_{4}$ PFUdA & - & - & - \\
\hline${ }^{13} \mathrm{C}_{2}$ Perfluoro-dodecanoic acid & ${ }^{13} \mathrm{C}_{2}$ PFDoA & - & - & - \\
\hline${ }^{18} \mathrm{O}_{2}$ Perfluoro-hexanesulfonate & ${ }^{18} \mathrm{O}_{2}$ PFHxS & - & - & - \\
\hline${ }^{13} \mathrm{C}_{4}$ Perfluoro-octanesulfonate & ${ }^{13} \mathrm{C}_{4}$ PFOS & - & - & - \\
\hline${ }^{13} \mathrm{C}_{8}$ Perfluoro-1-octanesulfonamide & ${ }^{13} \mathrm{C}_{8}$ FOSA & - & - & - \\
\hline $\begin{array}{l}{ }^{2} \mathrm{H}_{7} \text { 2-N-methylperfluoro-1- } \\
\text { octanesulfonamido-ethanlo }\end{array}$ & ${ }^{2} \mathrm{H}_{7} N$-MeFOSE & - & - & - \\
\hline $\begin{array}{l}{ }^{2} \mathrm{H}_{9} \text { 2- } N \text {-ethylperfluoro-1- } \\
\text { octanesulfonamido-ethanlo }\end{array}$ & ${ }^{2} \mathrm{H}_{9} N$-EtFOSE & - & - & - \\
\hline
\end{tabular}

through a 2-mm mesh, and stored in pre-cleaned glass jars in room temperature until further analysis.

\section{Pretreatment}

Extraction of PFASs was performed by use of a previously published method with some modifications (Wang et al. 2013). A sample of $0.2 \mathrm{~g}$ of unextracted soil was analyzed directly for determining the concentrations of TF by using combustion ion chromatography (CIC). About $2.5 \mathrm{~g}$ soil sample was weighed and then transferred to a $50-\mathrm{mL}$ PP centrifuge tube along with $2 \mathrm{~mL}$ nano-pure water and vortexed. One milliliter of $0.5 \mathrm{M}$ TBAHS, $2 \mathrm{~mL}$ of $25 \mathrm{mM}$ sodium carbonate, and $1 \mathrm{ng}$ mass-labeled internal standards were added before extraction by shaking at $700 \mathrm{rpm}$ for $5 \mathrm{~min}$. Subsequently, $5 \mathrm{~mL}$ MTBE was added and shaken for a further $20 \mathrm{~min}$ at $400 \mathrm{rpm}$ and centrifuged for $20 \mathrm{~min}$ at $1,300 \times \mathrm{g}$. The $5 \mathrm{~mL}$ supernatant was transferred to a $15 \mathrm{~mL}$ PP tube and the extraction was repeated twice as described above, resulting in a final volume of $15 \mathrm{~mL}$ supernatant. The supernatant was evaporated to dryness under a gentle flow of high-purity nitrogen, and re-dissolved in $1 \mathrm{~mL}$ methanol. Of this solution, $0.2 \mathrm{~mL}$ was taken for quantification of EOF by combustion ion chromatography (CIC). The remaining $0.8 \mathrm{~mL}$ was mixed with $40 \mathrm{mg}$ Envi-carb and $1 \mathrm{~mL}$ methanol, followed by sonication for $30 \mathrm{~s}$ and centrifuging at $2,700 \times g$ for $20 \mathrm{~min}$. The supernatant was collected and 
Fig. 1 Soil sampling area and sites in Koshi River Watershed

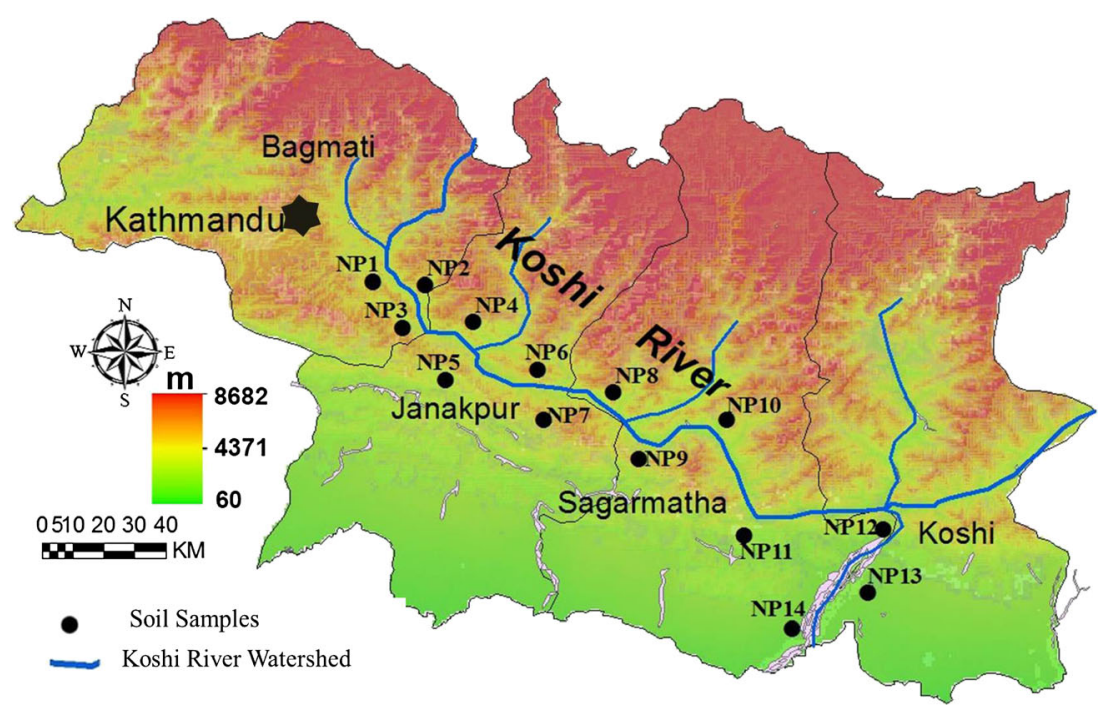

transferred to a new 15-mL PP tube. The remnant was rinsed twice with $1 \mathrm{~mL}$ methanol, followed by sonication and centrifuging, and the supernatant was combined together. Then the solution was reduced to $1 \mathrm{~mL}$ under nitrogen flow and filtered into a $1.5 \mathrm{~mL}$ PP GC vial by a $13 \mathrm{~mm} / 0.2-\mu \mathrm{m}$ nylon filter (Pall Corporation, New York, USA).

\section{TF and EOF analysis}

Samples were combusted in an AQF-100 automatic quick furnace, collected in a GA100 Gas Absorption Unit, and measured on a Dionex CD20 Conductivity Detector, using sodium fluoride as a standard solution with a calibration range for $\mathrm{F}^{-}$at 1 to $100 \mathrm{mg} \mathrm{L}^{-1}$. Concentrations of TF and EOF detected here are presented as corresponding concentrations of $\mathrm{F}^{-}\left(C_{\mathrm{F}}\right.$ in nanogram per gram). For comparison, concentrations of $C_{\mathrm{F}}$ were calculated from concentrations of individual PFASs using Eq. (1):

$C_{\mathrm{F}}=\frac{n M_{\mathrm{F}}}{M_{\mathrm{PFC}}} C_{\mathrm{PFC}}$

Where: $C_{\mathrm{PFC}}$ is concentration of PFC (in nanogram per gram dry dry weight), $M_{\mathrm{PFC}}$ is the molecular weight of corresponding PFC (in gram per mole), $n$ is the number of fluorines in the compound, and $M_{\mathrm{F}}$ is the molecular weight of fluorine (in gram per mole).

Identification and quantification of PFASs by HPLC-MS/MS

High-pressure liquid chromatography (HPLC) fitted with a Beatacil C18 column (Thermo Scientific, $100 \times 2.1 \mathrm{~mm}, 5-\mu \mathrm{m}$ particle size) was used for separation of analytes. Two millimolars of ammonium acetate was used as an ionization aid. Water and methanol were used as mobile phases. The temperature of the column oven was kept constant at $35^{\circ} \mathrm{C}$. Mass spectra were measured by a tandem mass spectrometer fitted with an electrospray ionization source (Applied Biosciences SCIEX 3000) operating in negative ionization mode with chromatograms recorded using multiple reaction monitoring (MRM) mode. A desolation temperature was set at $450{ }^{\circ} \mathrm{C}$, ion spray voltage was $-3,500 \mathrm{~V}$, collision gas set at 12 arbitrary units (AU) with curtain gas set at $6 \mathrm{AU}$, nebulizer gas was set to $5 \mathrm{AU}$ and dwell times of $40 \mathrm{~ms}$. Quantification was done by use of Analyst 1.4.1 software (SCIEX, Applied Biosciences Foster City, CA).

Quality control and quality assurance

For quality assurance and quality control, procedural blanks, and matrixes spiked with the standard solution were analyzed. Use of Teflon-coated lab-ware was avoided during all steps of sample preparation and analysis to minimize contamination. Concentrations of 16 PFASs were quantified by use of 7 points internal calibration curves containing a concentration series from 0.01 to $10 \mathrm{ng} / \mathrm{mL}$ for native standards and a constant concentration of $1 \mathrm{ng} / \mathrm{mL}$ for mass-labeled standards. Concentrations of all target PFASs in all field and laboratory blanks were lower than the detection limit (LOD), which was defined as a signal-tonoise ratio of 3:1. Recoveries of PFASs ranged from $75 \pm 8$ to $107 \pm 12 \%$, and the LOD was from 0.03 to $0.30 \mathrm{ng} / \mathrm{g}$ (Table 1).

\section{Results and discussion}

PFASs in soils around the Koshi River

Among the 16 PFASs investigated, only 10 were detected (Table 2). Concentrations of perfluoroalkyl carboxylic acids (PFCAs) ranged from nd (below the detection limit) to $1.48 \mathrm{ng} / \mathrm{g} \mathrm{dw}$, while concentrations of perfluoroalkyl sulfonic 
Table 2 Concentrations of PFASs, TF, EOF in soils around the Koshi River (in nanogram per gram dry weight)

\begin{tabular}{|c|c|c|c|c|c|c|c|c|c|c|c|c|c|c|}
\hline \multirow[t]{2}{*}{ Sample ID } & \multicolumn{6}{|c|}{ PFCAs } & \multicolumn{2}{|c|}{ PFSAs } & \multicolumn{2}{|l|}{ Precursors } & \multirow[t]{2}{*}{ PFASs } & \multirow[t]{2}{*}{ PFASs_F } & \multirow[t]{2}{*}{ EOF } & \multirow[t]{2}{*}{$\mathrm{TF}$} \\
\hline & PFBA & PFPeA & PFHxA & PFHpA & PFOA & PFDoA & PFBS & PFOS & $N$-MeFOSE & $N$-EtFOSE & & & & \\
\hline NP1 & nd & nd & 0.12 & nd & nd & nd & 0.12 & nd & nd & nd & 0.24 & 0.14 & 172.18 & 560,955 \\
\hline NP2 & nd & nd & nd & nd & nd & nd & nd & nd & nd & nd & 0.00 & 0.00 & 143.56 & 674,214 \\
\hline NP3 & nd & nd & nd & nd & nd & nd & nd & nd & nd & nd & 0.00 & 0.00 & 143.00 & 393,181 \\
\hline NP4 & nd & nd & nd & nd & 0.10 & nd & nd & nd & 0.63 & nd & 0.73 & 0.43 & 116.02 & 416,409 \\
\hline NP5 & 0.58 & nd & nd & nd & 0.26 & nd & nd & nd & nd & nd & 0.84 & 0.54 & 81.43 & 477,370 \\
\hline NP6 & nd & 0.20 & nd & nd & 0.13 & nd & 0.25 & nd & nd & nd & 0.58 & 0.35 & 137.80 & 705,684 \\
\hline NP7 & nd & nd & nd & nd & 0.12 & nd & nd & nd & nd & 0.35 & 0.47 & 0.28 & 137.02 & 550,487 \\
\hline NP8 & nd & nd & 0.14 & nd & nd & nd & 0.20 & nd & nd & nd & 0.34 & 0.19 & 116.77 & 666,580 \\
\hline NP9 & 0.41 & nd & nd & nd & 0.13 & nd & nd & nd & nd & nd & 0.54 & 0.34 & 153.92 & 614,139 \\
\hline NP10 & 0.54 & nd & nd & nd & 0.20 & 0.25 & 0.38 & 0.13 & nd & nd & 1.51 & 0.93 & 200.18 & 738,672 \\
\hline NP11 & nd & 0.35 & 0.22 & 0.78 & 0.13 & nd & nd & nd & nd & 0.30 & 1.78 & 1.16 & 68.92 & 662,254 \\
\hline NP12 & 0.46 & nd & 0.16 & nd & nd & nd & nd & 0.10 & nd & 0.34 & 1.06 & 0.64 & 99.32 & 335,099 \\
\hline NP13 & nd & nd & 0.10 & nd & 0.12 & nd & 0.18 & nd & nd & nd & 0.40 & 0.24 & 35.24 & 513,059 \\
\hline NP14 & nd & nd & nd & nd & nd & nd & 0.29 & nd & nd & nd & 0.29 & 0.15 & 198.96 & 362,645 \\
\hline
\end{tabular}

$n d$ below the LOD, PFASs_F concentrations of PFASs presented as corresponding concentrations of $\mathrm{F}^{-}$

acids (PFSAs) ranged from nd to $0.51 \mathrm{ng} / \mathrm{g} \mathrm{dw}$. The precursors of PFOS, $N$-MeFOSE was detected only in NP4 $(0.63 \mathrm{ng} / \mathrm{g}$ $\mathrm{dw}$ ), while $N$-EtFOSE were detected in three sites (NP7, NP11, NP13), ranging from concentrations less than nd to $0.35 \mathrm{ng} / \mathrm{g} \mathrm{dw}$, while the other precursors were below the LOD in all sites. Detection ratio of PFOA (57 \%), PFBS (43\%), PFHxA (36 \%), PFBA (29 \%) were relatively high. PFHpA was found exclusively in NP11 and PFDoA was found exclusively in NP10, they were with the least rate of detection of the PFASs. PFCAs were the predominant PFASs found in soils, with relatively high percentages of $100 \%$ in NP5, and $83 \%$ in NP11. Currently, there are very few regulations regarding PFASs in soils developed to protect soil organisms and human health. Concentrations of PFOA and PFOS were significantly less than the residential soil screening guidance values issued by US-EPA (PFOA, 16,000 ng/g; PFOS, 6,000 ng/g) (USEPA 2009). Results obtained from the present study were compared with those previously reported in other areas (Table 3) (Wang et al. 2011; Wang et al. 2012a; Meng et al. 2013a; Naile et al. 2010; Li et al. 2010). Basically, Concentrations of PFASs around the Koshi River were less than those reported from the areas with higher level of industrialization, such as Shanghai, Tianjin Binhai New Area in Chin and the west coast of South Korea. Different levels of industrialization may account for this phenomenon. Nepal is a typical agricultural country with a slower process of industrialization and urbanization than other regions cited in this study. These observed results are in agreement with the previous observation of PFASs in water, which revealed a possible relationship between concentrations of PFASs and the levels of industrialization (Wang et al. 2012b).

Spatial pattern of PFASs in soils around the Koshi River

Concentrations of PFASs in soils were relatively evenly distributed among samples of soils with slightly higher concentrations in downstream (Fig. 2). Maximum concentrations of PFASs occurred in NP11 (1.78 ng/g dw), which was close to

Table 3 Comparison of PFASs concentrations in soils from different study areas

\begin{tabular}{lllllll}
\hline & Location (sample size) & $\begin{array}{l}\text { Sampling } \\
\text { time }\end{array}$ & PFOA & PFOS & PFASs & References \\
\hline Nepal & Koshi River (14) & 2010 & nd-0.26 & nd-0.13 & nd-1.78 & Present study \\
China & Guanting Reservoir (7) & 2008 & nd-2.8 & nd-0.86 & $0.13-8.5$ & Wang et al. (2011) \\
& Huaihe River (18) & 2008 & nd-0.20 & nd-0.21 & nd-1.22 & Meng et al. (2013a) \\
& Tianjin Binhai New Area (8) & 2008 & nd-0.93 & nd-9.4 & $1.3-11$ & Wang et al. (2012a, b) \\
& Shanghai (8) & 2007 & $3.28-47.5$ & $8.58-10.4$ & $14.41-63.62$ & Li et al. (2010) \\
South Korea & Western Coast (13) & 2009 & nd-3.4 & nd-1.7 & $0.28-3.9$ & Naile et al. (2010) \\
\hline
\end{tabular}


Fig. 2 Spatial distribution and relative contributions of individual PFASs in soils along the Koshi River

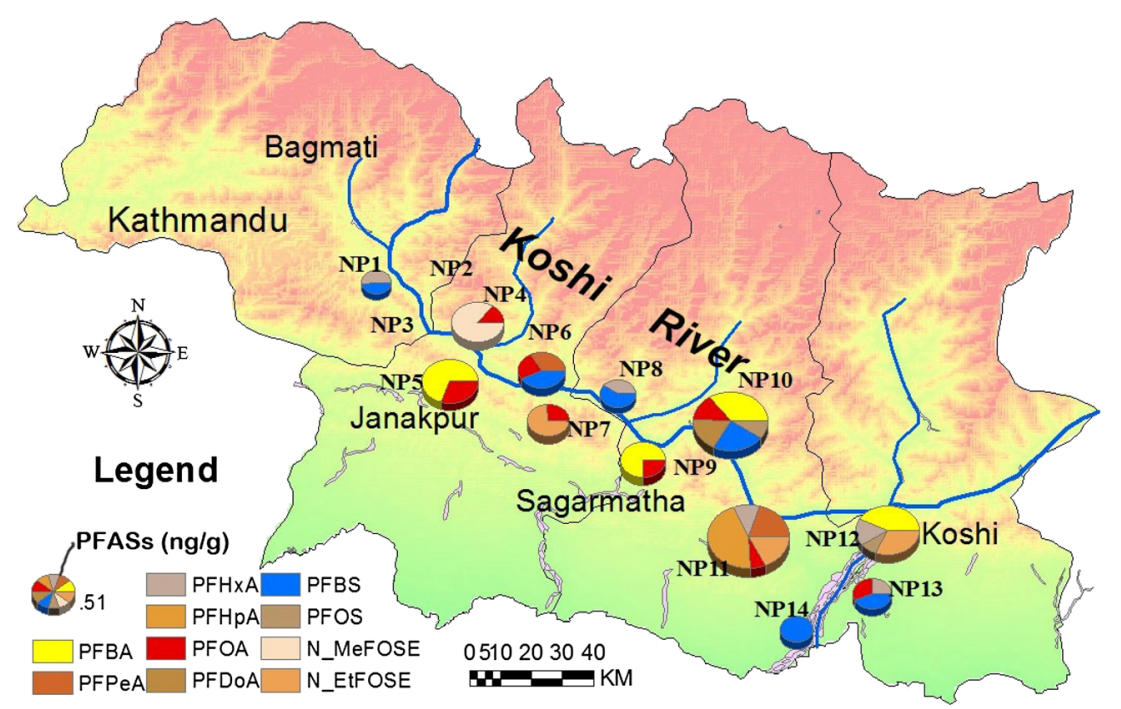

the road and had been easily affected by anthropogenic activity, particularly, transportation, and rubbish dump. Site NP10, with a total PFASs concentration of $1.51 \mathrm{ng} / \mathrm{g} \mathrm{dw}$ was once a palace and landslides often happened here. Concentrations of all investigated PFASs in location of NP2 and NP3 were less than the LOD. This is probably because these regions are remote from villages avoiding the intensive anthropogenic activities.

Relative concentrations of individual PFASs varied among locations. The compositions of more detectable PFASs observed during this study, PFOA, with a detection rate of $62 \%$, varied from $7 \%$ (NP11) to $31 \%$ (NP5), and those of PFBS with a detection rate of $46 \%$ varied from $5 \%$ (NP10) to $100 \%$ (NP14) at their detected locations, respectively. PFHxA and PFBA, with relatively greater rates of detection of 36 and $29 \%$, accounted for percentages of $12 \%$ (NP11) to $52 \%$ (NP1) for PFHxA and $36 \%$ (NP10) to $76 \%$ (NP5) for PFBA at their detected sites, respectively. Contributions of PFOS, a widespread PFASs in most studies (Li et al. 2010; Naile et al. 2010; Meng et al. 2013a), while having a lesser rate of detection of $14 \%$ in this study, ranged from nd to $9 \%$ (NP12). This result might be due to the development of corresponding substitutes in current years, mostly known as PFBA and PFBS (Wang et al. 2013). In addition, each PFASs showed a relatively balanced distribution, which may imply that they are from different sources.

\section{Relationship between PFASs and land use}

Based on type of soil cover, samples were grouped into woodland $(n=5)$, maize land $(n=6)$, vegetable land $(n=2)$, and paddy ( $n=1)$ (Fig. 3 and Table S2). The mean concentration of PFASs in woodland soils $(0.96 \mathrm{ng} / \mathrm{g} \mathrm{dw})$ was generally higher than those in farmland soils $(0.44 \mathrm{ng} / \mathrm{g} \mathrm{dw})$, including those used to grow maize, vegetables, and paddy. The woodland soils were covered with thick leaves, thus resulting in sufficient organic matter under the relative warm and humid climate. Woodland soils also have a higher scavenging power of atmospheric pollutants, which they eventually deposit in soils. This is especially true for locations NP11, NP10, and NP5, which were located in hills and covered with dense forests. Woodland soils tended to remain stable and nonploughed, while farmland was subjected to regular plough and rotation, which could speed up the degradation of PFASs. With respect to the different types of crops, concentrations of PFASs in maize land soils exhibited an even distribution except for location of NP2. In the paddy field of NP12, PFASs also had a higher concentration, and lower concentrations of PFASs were found in soils where vegetables were grown. Although PFASs in woodland soils showed general higher concentrations than those in farmland, no obvious evidence could prove that emissions of PFASs were closely related to land use, according with previously reported results conducted in the Huaihe River (Meng et al. 2013b). Due to the limited number of samples in present study, it was not possible to draw certain conclusions about the relationship between PFASs and land use. As a result, more detailed research, such as the analysis of soil profiles, more specific land use division, and intensive sampling around the river, is required to further determine the possible relationship between PFASs distribution and different land use. Meanwhile, questionnaires among the villages about the historical changes of land use types are also needed to identify the relationship more accurately.

Principal component analysis

Patterns of distribution of relative concentrations of organic Carbon (OC), pH, individual PFASs, EOF, and TF in soil were determined by use of principal components analysis (PCA). 
Fig. 3 Concentrations of PFASs in soils from different land use types (in nanogram per gram dry dry weight)

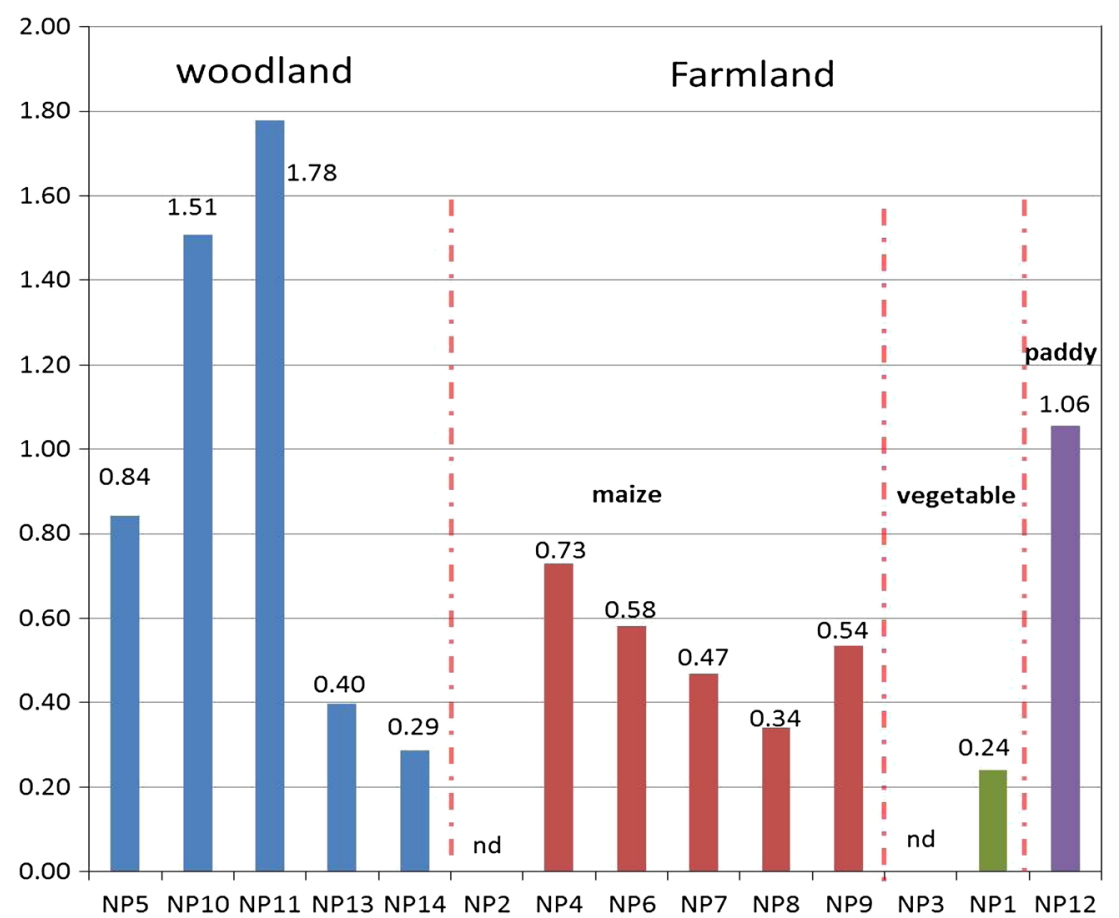

The components were ranked by their eigenvalues (Table S3). The first three components explained nearly $80 \%$ of total variance. Analysis of the PCA indicated that the distribution pattern of the most frequently detected PFASs: PFOA and PFBS, only PFOA was associated with OC contents of soils in the Koshi River (Table S4 and Fig. S1). Previous studies have revealed that sorption of PFASs onto sediments is strongly correlated with OC fraction (Higgins and Luthy 2006) and the average log Koc was directly proportional to number of carbons in PFCAs (Labadie and Chevreuil 2011; Ahrens et al. 2009). In this study, the limited detection of other PFASs restricted the ability to examine this relationship between $\mathrm{OC}$ and PFASs distribution.

\section{Potential sources of PFASs around the Koshi River}

Given the different but relatively balanced distribution of each PFASs and the uncertain relationship between PFASs and land use, it is hard to identify the specific industrial or agricultural source of PFASs around the Koshi River in the present study. In order to infer the possible source of PFASs, it should be noted that because of the absence of direct PFASs manufacturing in Nepal and PFASs-related chemical plants located around the sampling sites, PFASs in soil are mainly from the long-range atmospheric transport. PFASs-related chemical applications, domestic consumption, and disposal of PFASscontaining products were generally thought to be the potential sources. Previous researches have elucidated that PFASs in soils partly come from atmospheric transport, possibly (Kim and Kannan 2007; Simcik and Dorweiler 2005). Precursors of
PFASs, such as fluorotelomer alcohol (FTOH), perfluoroalkyl sulfonamide, and their atmospherically oxidative products, existing in air are washed down by precipitation, and then absorbed by soil (Kim and Kannan 2007; Liu and Lee 2007). Furthermore, some PFASs existing in house and street dust could also be absorbed by soil and contribute to their levels in soils (Strynar and Lindstrom 2008; Murakami and Takada 2008). Nepal borders with India and China, where PFASs are heavily applied and manufactured. Previous studies have assessed that long-range transport is greater for longer-chain PFASs (Armitage et al. 2009), so it shouldn't be neglected that the neighboring countries' applications of PFASs are also the possible source of PFASs occurrence in Nepal. As to the domestic industrial application, in central and eastern Nepal there are some industries where PFASs are widely used. These include textile treatment, leather shoes, carpet, food processing paper, and so on, which are thought to produce PFASsrelated chemicals releases during applications (Xie et al. 2013).

Another non-ignorable potential source is domestic consumption and disposal of PFASs-containing products. Uncontrolled disposal of PFASs-containing products, for example, worn carpet, clothes, leather, or discarded food packaging, can cause PFASs to be released into the environment. One of the prominent phenomena in this study is the relatively high detection rates of short-chain PFASs, especially PFBA and PFBS. With reductions in manufacture of PFOA and PFOS, the fluoropolymer industry has been using these $\mathrm{C} 4$ PFASs in place of PFOS and PFOA, which has resulted in widespread contamination of the environment with these 
Fig. 4 Spatial distribution and relationship of individual PFASs $\left(\mathrm{C}_{\mathrm{F}}\right)$ and EOF in soils along the Koshi River

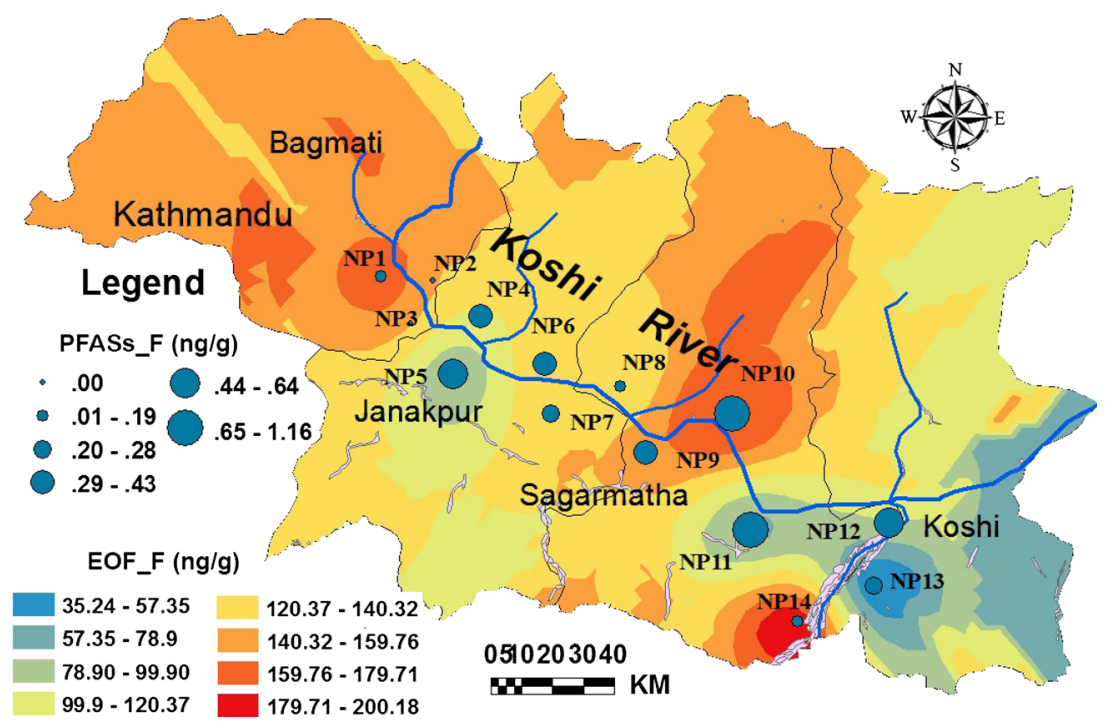

chemicals (Wang et al. 2013). Since Nepal is a developing country, reports of improper waste management, specifically, direct waste dump to roadside and riverbank (Pokhrel and Viraraghavan 2005) could contribute to PFASs emission. In spite of the difficulty in source identification, sufficient attention should be around. Effective measures should be taken into consideration, for example, statistics of specific industrial distribution and application of PFASs-related chemicals, and calculation of industrial and domestic emission, what's more, reinforcement of waste disposal management.

Mass balance of fluorine in soils

Mass balance analysis of fluorine has been widely applied to explain PFASs distribution, transport, and environmental behaviors (Miyake et al. 2007; Yeung et al. 2008). Total concentrations of PFASs, expressed as equivalent concentrations of fluorine, ranged from nd to $1.16 \mathrm{ng} / \mathrm{g} \mathrm{dw}$, while concentrations of EOF ranged from 35.2 to $200.2 \mathrm{ng} / \mathrm{g} \mathrm{dw}$ and concentrations of $\mathrm{TF}$ ranged from 335,099 to $738,672 \mathrm{ng} / \mathrm{g} \mathrm{dw}$, respectively (Table 2; Figs. 4 and 5). These results indicate that EOF accounted for a small proportion of TF in soils with percentages from 0.01 to $0.05 \%$ (average, $0.02 \%$ ), while inorganic fluorine (IF) and non-extractable fluorine (NEOF) made up the major portion of TF. The same trend was observed previously for soil (Wang et al. 2013), water (Miyake et al. 2007), human blood (Yeung et al. 2008), and livers of marine mammals (Yeung et al. 2009b).

Spatial distribution relationships between PFASs and EOF, EOF, and TF were analyzed to illustrate environmental behaviors of fluorine in different forms (Figs. 4 and 5). There was an obvious trend that EOF in NP10 (200.2 ng/g dw), NP14 (199.0 ng/g dw), NP1 (172.2 ng/g dw) occurred at relatively high concentrations. While among these sites,
Fig. 5 Spatial distribution and relationship of individual EOF and $\mathrm{TF}$ in soils along the Koshi River

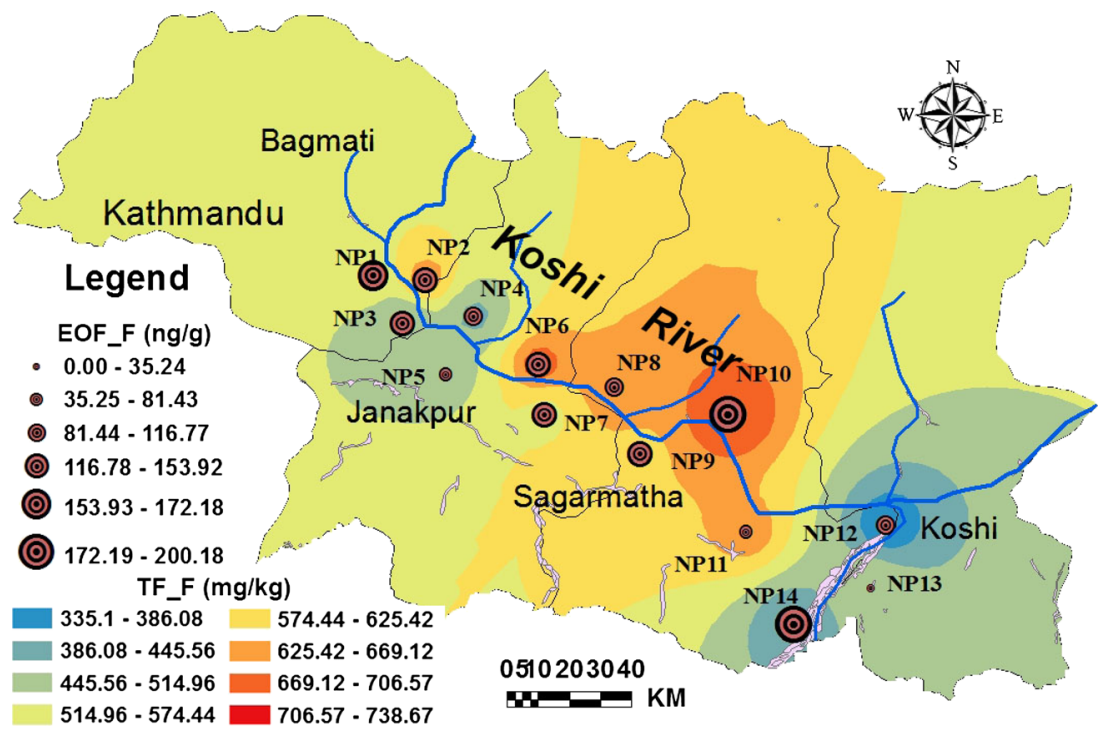


higher concentration of PFASs was only found in NP10 $\left(0.93 \mathrm{ng} \mathrm{F}^{-} / \mathrm{g}\right)$. The highest concentrations of PFASs was observed at location NP11 (1.16 $\left.\mathrm{ng} \mathrm{F}^{-} / \mathrm{g}\right)$, where a lower concentration of EOF $(68.9 \mathrm{ng} / \mathrm{g} \mathrm{dw})$ was observed. In the sites with no detected PFASs in NP2 and NP3, while concentrations of EOF were found at relatively high concentrations of 143.6 and $143.0 \mathrm{ng} / \mathrm{g} \mathrm{dw}$, respectively. In brief, the spatial distribution of EOF and PFASs did not present a very significant correlation only with a slightly negative correlation $\left(\gamma_{\text {PFASs-EOF }}=-0.374\right)$, which may be attributed to the low contributions of PFASs to EOF ranging from 0 to $1.68 \%$ (average, $0.39 \%$ ).

In terms of TF and EOF, the results of this study were comparable to those of previous studies of soils in the region around Liaodong Bay (Wang et al. 2013) and the Huaihe River, China (Meng et al. 2013b). Concentrations of TF and EOF in Liaodong Bay ranged from 219,468 to $738,504 \mathrm{ng} / \mathrm{g}$ $\mathrm{dw}$ and 30.8 to $208.5 \mathrm{ng} / \mathrm{g} \mathrm{dw}$, respectively. Those in the region of the Huaihe River ranged from 270,117 to $949,866 \mathrm{ng} / \mathrm{g} \mathrm{dw}$ and 67.4 to $225.5 \mathrm{ng} / \mathrm{g} \mathrm{dw}$, respectively. Concentrations of TF and EOF in soils did not show significant variation in different regions, which is possibly because fluorine is a basic element of natural soil and the occurrence of PFASs and EOF is a just small portion of the total fluorine content. According to study of Wang et al. (2013), weak correlations among TF, EOF, and PFASs $\left(\gamma_{\text {PFASs-EOF }}\right.$ $=0.013, \gamma_{\text {EOF-TF }}=0.301$ ) were also reported, indicating that no consistency in the pattern of spatial distributions of TF, EOF, and PFASs in soils, which can be explained that owing to the complexity of soil texture. PFASs and EOF are more likely to be absorbed into soils or transformed into other forms. Furthermore, the complicated and various sources of PFASs also increase the difficulty of determining their distribution features.

Recent observations conducted in soils obtained different results compared with those in biota where relatively large contributions of PFASs/EOF and EOF/TF were observed. In particular, the contributions of PFASs/EOF and EOF/TF were 29.6 and $58.3 \%$ in livers of dolphins and 30.8 and $27.3 \%$ in those of finless porpoises (Yeung et al. 2009b). Furthermore, a study in a nature reserve in Hong Kong also found that ranges of contributions of PFASs/EOF and EOF/TF were 40.87$75.76 \%$ and $29.49-43.46 \%$ in livers of different fishes (Loi et al. 2011). In human blood, the contributions of PFASs/EOF and EOF/TF were higher up to 85 and $40.4 \%$ (Yeung et al. 2008). In contrast, the composition of PFASs/EOF and EOF/ TF in soils was relatively small, with average proportions of 0.39 and $0.02 \%$ in the present study, and 0.77 and $0.02 \%$ in Liaodong Bay of China (Wang et al. 2013). These are lesser than $36.02 \%$ of PFASs/EOF and $0.10 \%$ of EOF/TF in contaminated seawater, as well as $0.89 \%$ of PFASs/EOF and $0.01 \%$ of EOF/TF in considered non-pollution seawater (Miyake et al. 2007). In addition to the complex texture of soils, the unknown or unanalyzed PFASs in soils also impact their accurate quantitation. Methods used to extract OF varies among studies and the optimum method has not yet been established is with controversy (Yeung et al. 2009b).

\section{Conclusions}

Among the 16 PFASs investigated, 10 compounds were detected with PFOA, PFBS, and PFBA being predominant in soils along the Koshi River in Nepal. Concentrations of PFASs ranged from $<$ LOD to $1.78 \mathrm{ng} / \mathrm{g} \mathrm{dw}$ which were lesser than those observed in more developed regions. Concentrations of PFASs were higher in those regions with more human activities. Since they are not produced locally PFASs in soils in Nepal likely came from use and disposal of PFASs-containing products in addition to long range atmospheric transport and deposition. Mass balance analysis indicated that there was limited correlation among PFASs, EOF, and $\mathrm{TF}$, which is probably due to the multiple sources and irregularity in distributions of PFASs in soils. Nevertheless, PFBS and PFBA, which are being used as substitutes for PFOS and PFOA, which are being phased out of many uses have been widely spread in remote and less developed areas. Even though these shorter chair replacements are less accumulated in biota and have lesser toxic potencies, because they are persistent, more attention to those short-chain PFASs. Baseline information on PFASs was provided on Nepal for the first time. Further work is strongly needed to determine fate and potential risk of PFASs in soils. Effective measures, for example, statistics of industrial and domestic emission and reinforcement of waste disposal management by Nepalese government, are also recommended to undertake in order to control PFASs contamination.

Acknowledgements This study was supported by the National Natural Science Foundation of China under grant no. 41171394, the Key Research Program of the Chinese Academy of Sciences under grant no. KZZD-EW-TZ-12, and the Strategic Priority Research Program of the Chinese Academy of Sciences with grant no. XDB03030504. Portions of the research were supported by a Discovery Grant from the National Science and Engineering Research Council of Canada (project no. 326415-07). Professor Giesy was supported by the Canada Research Chair Program. Finally, we would like to thank the editors and reviewers for their valuable comments and suggestions.

\section{References}

Ahrens L, Yamashita N, Yeung LWY, Taniyasu S, Horii Y, Lam PKS, Ebinghaus R (2009) Partitioning behavior of per- and polyfluoroalkyl compounds between pore water and sediment in two sediment cores from Tokyo Bay, Japan. Environ Sci Technol 43(18):6969-6975 
Aichner B, Glaser B, Zech W (2007) Polycyclic aromatic hydrocarbons and polychlorinated biphenyls in urban soils from Kathmandu, Nepal. Org Geochem 38(4):700-715

Armitage JM, MacLeod M, Cousins IT (2009) Comparative assessment of the global fate and transport pathways of long-chain perfluorocarboxylic acids (PFCAs) and perfluorocarboxylates (PFCs) emitted from direct sources. Environ Sci Technol 43(15): $5830-5836$

Bhatt MP, Gardner KH (2009) Variation in DOC and trace metal concentration along the heavily urbanized basin in Kathmandu Valley, Nepal. Environ Geol 58(4):867-876

Corsolini S, Sarkar SK, Guerranti C, Bhattacharya BD, Rakshit D, Jonathan MP, Godhantaraman N (2012) Perfluorinated compounds in surficial sediments of the Ganges River and adjacent Sundarban mangrove wetland, India. Mar Pollut Bull 64(12):2829-2833

Dahal BM, Fuerhacker M, Mentler A, Karki KB, Shrestha RR, Blum WEH (2008) Arsenic contamination of soils and agricultural plants through irrigation water in Nepal. Environ Pollut 155(1):157-163

Dreyer A, Weinberg I, Temme C, Ebinghaus R (2009) Polyfluorinated compounds in the atmosphere of the Atlantic and Southern Oceans: evidence for a global distribution. Environ Sci Technol 43(17): $6507-6514$

Giesy JP, Kannan K (2001) Global distribution of perfluorooctane sulfonate in wildlife. Environ Sci Technol 35(7):1339-1342

Giesy JP, Kannan K (2002) Perfluorochemical surfactants in the environment. Environ Sci Technol 36(7):146A-152A

Guzzella L, Poma G, De Paolis A, Roscioli C, Viviano G (2011) Organic persistent toxic substances in soils, waters and sediments along an altitudinal gradient at Mt. Sagarmatha, Himalayas, Nepal. Environ Pollut 159(10):2552-2564

Hansen KJ, Johnson HO, Eldridge JS, Butenhoff JL, Dick LA (2002) Quantitative characterization of trace levels of PFOS and PFOA in the Tennessee River. Environ Sci Technol 36(8):1681-1685

Higgins CP, Luthy RG (2006) Sorption of perfluorinated surfactants on sediments. Environ Sci Technol 40(23):7251-7256

Karrman A, Ericson I, van Bavel B, Darnerud PO, Aune M, Glynn A, Lignell S, Lindstrom G (2007) Exposure of perfluorinated chemicals through lactation: levels of matched human milk and serum and a temporal trend, 1996-2004, in Sweden. Environ Health Perspect $115(2): 226-230$

Kim SK, Kannan K (2007) Perfluorinated acids in air, rain, snow, surface runoff, and lakes: relative importance of pathways to contamination of urban lakes. Environ Sci Technol 41(24):8328-8334

Kissa E (2001) Fluorinated surfactants and repellents, 2nd edn. Marcel Decker Inc., New York

Labadie P, Chevreuil M (2011) Partitioning behaviour of perfluorinated alkyl contaminants between water, sediment and fish in the Urge River (nearby Paris, France). Environ Pollut 159(2):391-397

Li F, Zhang CJ, Qu Y, Chen J, Chen L, Liu Y, Zhou Q (2010) Quantitative characterization of short- and long-chain perfluorinated acids in solid matrices in Shanghai, China. Sci Total Environ 408(3):617623

Liu JX, Lee LS (2007) Effect of fluorotelomer alcohol chain length on aqueous solubility and sorption by soils. Environ Sci Technol 41(15):5357-5362

Loi EIH, Yeung LWY, Taniyasu S, Lam PKS, Kannan K, Yamashita N (2011) Trophic magnification of poly- and perfluorinated compounds in a subtropical food web. Environ Sci Technol 45(13): 5506-5513

Meng J, Wang TY, Wang P, Giesy JP, Lu YL (2013a) Perfluorinated compounds and organochlorine pesticides in soils around Huaihe River: a heavily contaminated watershed in Central China. Environ Sci Pollut Res 20(6):3965-3974

Meng J, Wang TY, Wang P, Lu YL (2013b) Spatial distribution and composition of perfluorinated compounds in soils around the Huaihe River. Environ Sci 34(8):3187-3194 (in Chinese)
Miyake Y, Yamashita N, Rostkowski P, So MK, Taniyasu S, Lam PKS, Kannan K (2007) Determination of trace levels of total fluorine in water using combustion ion chromatography for fluorine: a mass balance approach to determine individual perfluorinated chemicals in water. J Chromatogr A 1143(1-2):98-104

Murakami M, Takada H (2008) Perfluorinated surfactants (PFSs) in sizefractionated street dust in Tokyo. Chemosphere 73(8):1172-1177

Naile JE, Khim JS, Wang TY, Chen CL, Luo W, Kwon BO, Park J, Koh CH, Jones PD, Lu YL, Giesy JP (2010) Perfluorinated compounds in water, sediment, soil and biota from estuarine and coastal areas of Korea. Environ Pollut 158(5):1237-1244

NPHC (2012) National Population and Housing Census 2011 (National Report). Central Bureau of Statistics, Nepal

Oliaei F, Kriens D, Weber R, Watson A (2013) PFOS and PFC releases and associated pollution from a PFC production plant in Minnesota (USA). Environ Sci Pollut Res 20(4):1977-1992

Pico Y, Farre M, Llorca M, Barcelo D (2011) Perfluorinated compounds in food: a global perspective. Crit Rev Food Sci Nutr 51(7):605-625

Pokhrel D, Viraraghavan T (2005) Municipal solid waste management in Nepal: practices and challenges. Waste Manag 25(5):555-562

Shi YL, Pan YY, Yang RQ, Wang YW, Cai YQ (2010) Occurrence of perfluorinated compounds in fish from Qinghai-Tibetan Plateau. Environ Int 36(1):46-50

Shrestha SH (2005) Nepal in maps. Educational Publishing House, Kathmandu, Nepal

Simcik MF, Dorweiler KJ (2005) Ratio of perfluorochemical concentrations as a tracer of atmospheric deposition to surface waters. Environ Sci Technol 39(22):8678-8683

Strynar MJ, Lindstrom AB (2008) Perfluorinated compounds in house dust from Ohio and North Carolina, USA. Environ Sci Technol 42(10):3751-3756

US-EPA (2009) Soil Screening Levels for Perfluorooctanoic Acid (PFOA) and Perfluorooctyl Sulfonate (PFOS); Memorandum: Atlanta, GA. http://www.epa.gov/region4/water/documents/final pfc_soil_screening_values11_20_09.pdf

US-EPA (2013) Emerging Contaminants Fact Sheet - PFOS and PFOA $\mathrm{http} / / /$ www.epa.gov/fedfac/pdf/ec_technical_fs_pfos_pfoa_march 2013.pdf

Wang T, Wang YW, Liao CY, Cai YQ, Jiang GB (2009) Perspectives on the inclusion of perfluorooctane sulfonate into the Stockholm convention on persistent organic pollutants. Environ Sci Technol 43(14):5171-5175

Wang TY, Chen CL, Naile JE, Khim JS, Giesy JP, Lu YL (2011) Perfluorinated compounds in water, sediment and soil from Guanting Reservoir, China. Bull Environ Contam Toxicol 87(1):74-79

Wang TY, Lu YL, Chen CL, Naile JE, Khim JS, Giesy JP (2012a) Perfluorinated compounds in a coastal industrial area of Tianjin, China. Environ Geochem Health 34(3):301-311

Wang TY, Khim JS, Chen CL, Naile JE, Lu YL, Kannan K, Park JS, Luo W, Jiao WT, Hu WY, Giesy JP (2012b) Perfluorinated compounds in surface waters from Northern China: comparison to level of industrialization. Environ Int 42:37-46

Wang P, Wang TY, Giesy JP, Lu YL (2013) Perfluorinated compounds in soils from Liaodong Bay with concentrated fluorine industry parks in China. Chemosphere 91(6):751-757

Xie SW, Wang TY, Liu SJ, Jones KC, Sweetman AJ, Lu YL (2013) Industrial source identification and emission estimation of perfluorooctane sulfonate in China. Environ Int 52:1-8

Yamashita N, Kannan K, Taniyasu S, Horii Y, Petrick G, Gamo T (2005) A global survey of perfluorinated acids in oceans. Mar Pollut Bull 51(8-12):658-668

Yeung LWY, Miyake Y, Taniyasu S, Wang Y, Yu HX, So MK, Jiang GB, Wu YN, Li JG, Giesy JP, Yamashita N, Lam PKS (2008) Perfluorinated compounds and total and extractable organic fluorine in human blood samples from China. Environ Sci Technol 42(21): $8140-8145$ 
Yeung LWY, Yamashita N, Taniyasu S, Lam PKS, Sinha RK, Borole DV, Kannan K (2009a) A survey of perfluorinated compounds in surface water and biota including dolphins from the Ganges River and in other waterbodies in India. Chemosphere 76(1):55-62

Yeung LWY, Miyake B, Li P, Taniyasu S, Kannan K, Guruge KS, Lam PKS, Yamashita N (2009b) Comparison of total fluorine, extractable organic fluorine and perfluorinated compounds in the blood of wild and pefluorooctanoate (PFOA)-exposed rats: evidence for the presence of other organofluorine compounds. Anal Chim Acta 635(1): $108-114$

Young CJ, Furdui VI, Franklin J, Koerner RM, Muir DCG, Mabury SA (2007) Perfluorinated acids in arctic snow: new evidence for atmospheric formation. Environ Sci Technol 41(10):3455-3461 